\title{
Should the chairperson control IACUC meeting minutes?
}

Dr. Richard Cornish was the new chairperson of the Great Eastern University IACUC. Cornish believed that his primary responsibilities were to get protocols approved and support the school's faculty. He was always looking for shortcuts to approve protocols and ways to sidestep regulations he believed to be potentially deleterious to the faculty. Therefore, it did not surprise anybody on the committee that Cornish wanted to suppress the dissemination of information about a faculty member who received a written rebuke from the IACUC for telling his staff to ignore any critiques originating from the IACUC pre-review process and just submit the protocol as it was originally written. Cornish did not support the reprimand but it was nevertheless approved by the committee.

To prevent further harm to the faculty member, Cornish waited until the next IACUC meeting and then made a suggestion that seemed reasonable to some members. He proposed a policy that stated, "Minutes of IACUC meetings, including records of attendance, activities of the committee and committee deliberations shall be maintained by the IACUC. The chairperson shall review the minutes and append or redact any information therein judged to be omitted or superfluous." He explained that it was a federal requirement to maintain meeting minutes but there was no policy requiring the minutes to be approved by the IACUC, which had been the previous procedure. Therefore, he said, his suggested policy would save time and effort for the committee.

The IACUC administrator and one other member spoke up against the proposal, arguing that everybody should be privy to the minutes and only the full committee should be allowed to approve any alteration of the minutes. Cornish forcefully defended his position, noting that the minutes are posted on a secure internet site where anybody on the committee could read them. He quickly called for a vote and the new policy was approved.

Do you think that the new policy would be acceptable to the appropriate federal regulatory and oversight agencies? How would you approach this situation?

\section{RESPONSE}

\section{Flawed logic}

\section{Regina Correa-Murphy, BS, RLATG, CMAR}

Cornish seriously erred in thinking his primary responsibility as the IACUC chairperson was to get protocols approved. Although supporting the faculty seems noble at first glance, looking for shortcuts to approve protocols and ways to sidestep regulations might cause more harm than good to Great Eastern University's research program. If left unchecked, this behavior has the potential to draw intense scrutiny by regulatory oversight agencies and animal rights groups and might also attract negative publicity. The negative attention could ultimately result in the closure of or withdrawal of support for the animal program, hindering faculty members' research projects and their ability to obtain federal government funding.

Furthermore, Cornish's suppression of the information about a faculty member's reprimand was obstructive, especially if it involved a whistle-blower. This is a cause for concern for Great Eastern's leadership.

Cornish's policy allows him to use his personal and somewhat subjective view to determine what should be omitted or considered superfluous; this goes against the IACUC's primary responsibility: the assessment and oversight of the institution's animal program components and facilities ${ }^{1,2}$. Cornish's use of his position as the chairperson of the IACUC to alter policy that was approved by the duly composed IACUC by intimidation is coercive, and the forcing of a vote without allowing dissenting opinions to be acknowledged or even recorded can be considered an abuse of his role. Both the Animal Welfare Act ${ }^{2}$ and the Public Health Service Policy on Humane Care and Use of Laboratory Animals ${ }^{3}$ state that the IACUC minutes require records of attendance, activities of the committee and committee deliberations. Those minutes should provide sufficient detail, including dissenting opinions. Most institutions keep these records confidential by limiting access to IACUC members and oversight agencies that need to review and assess the animal care and use program.

Once this policy is reviewed by federal regulatory and other oversight agencies, Cornish and Great Eastern will have to answer some very difficult questions. Why are the minutes not reflective of the discussion including any dissenting IACUC members' views? What other shortcuts have been taken by investigators?

1. Institution for Laboratory Animal Research. Guide for the Care and Use of Laboratory Animals 8th edn. (National Academies Press, Washington, DC, 2011).

2. Animal Welfare Act. 9 CFR.

3. Public Health Service. Policy on Humane Care and Use of Laboratory Animals IV, E, 1, b (US Department of Health and Human Services, Washington, DC, 1986; amended 2002).

Correa-Murphy is Administrative Officer, Research and Development, Providence Veterans Affairs Medical Center, Providence, RI. 